\title{
Fabrication effects in the optical performance of DOEs engraved with femtosecond lasers
}

\author{
Angela Soria-Garcia ${ }^{\mathrm{a}}$, Jorge Fantova ${ }^{\mathrm{b}, \mathrm{c}}$, Alejandro San-Blas ${ }^{\mathrm{b}, \mathrm{c}}$, Jesus del Hoyo ${ }^{\mathrm{a}}$, Luis Miguel \\ Sanchez-Brea ${ }^{\mathrm{a}}$, Javier Alda ${ }^{\mathrm{d}}$, Ainara Rodriguez ${ }^{\mathrm{b}, \mathrm{c}}$, and Santiago Olaizola ${ }^{\mathrm{b}, \mathrm{c}}$ \\ ${ }^{a}$ Applied Optics Complutense Group, Optics Department, Universidad Complutense de \\ Madrid, Facultad de Ciencias Físicas, Plaza de las Ciencias 1, Madrid, 28040 Spain \\ ${ }^{\mathrm{b}}$ Ceit, Manuel Lardizabal 15, 20018 Donostia / San Sebastián, Spain \\ ${ }^{c}$ Universidad de Navarra, Tecnun, Manuel Lardizabal 13, 20018 Donostia / San Sebastián, \\ Spain \\ ${ }^{\text {d} A p p l i e d ~ O p t i c s ~ C o m p l u t e n s e ~ G r o u p, ~ U n i v e r s i d a d ~ C o m p l u t e n s e ~ d e ~ M a d r i d, ~ F a c u l t a d ~ d e ~}$ \\ Óptica y Optometría, Arcos de Jalón 118, Madrid, 28037 Spain
}

\begin{abstract}
The development of DOEs fabrication techniques is continuously growing due to the wide range of industrial applications, such as beam manipulation or optical position encoders. In this work, we use Femtosecond laser direct writing to manufacture DOEs, which uses a simpler and more efficient way to fabricate amplitude binary masks. Also, we have analyzed the performance of the DOEs. The fabrication technique is validated since the experimental results are in accordance to numerical simulations.
\end{abstract}

Keywords: Diffractive Optical Elements, laser recording, ultrashort laser, beam shaping, far-field diffraction, near-field diffraction.

\section{INTRODUCTION}

Diffractive Optical Elements (DOEs) are micro-structured devices which can modulate the amplitude, phase, or coherence polarization of a light beam. They are applied in multiple fields such as holography, ${ }^{1}$ beam shaping, generation of exotic beams ${ }^{2}$ or biomedicine. ${ }^{3}$ Over the years, the efficiency of DOEs' design algorithms have been increased, ${ }^{4}$ while fabrication methods are still under continuous study. ${ }^{5}$

Respect to design techniques, Gerchberg-Saxton algorithm is commonly used. It is a bidirectional iterative method which is used under far-field conditions and presents a high performance. ${ }^{6}$ On the other hand, several studies have been carried out to find out optimal fabrication techniques of DOEs. J. S. Sohn et al. ${ }^{7}$ propose a technique based on optical photolithography and A. Nottola et al. ${ }^{8}$ use a similar method, electron beam photolithography. Although the resolution achieved with these procedures is adequate, they are methods with several steps. Adam J. Caley et al. ${ }^{9}$ utilize a reactive ion etching technique, but again, it is not an easy way to produce DOEs.

We present a simpler method based on femtosecond laser direct writing ${ }^{10} \cdot{ }^{11}$ It has many advantages like a cleaner, quicker and contactless fabrication process which only requires a single step. Moreover, this method does not use pollutant chemicals, becoming one of the most environmentally friendly fabrication techniques. Amplitude modulation DOEs are implemented using a dielectric sample covered with a thin platinum layer (of a few hundred nanometers thick). Then, some parts of the metallic film are removed by laser ablation, resulting in a binary amplitude mask. In this way, DOEs may work both in transmission or reflection because the non-ablated metallic portions modulate both transmittance and reflectance with complementary spatial distributions.

With the aim of increasing the performance of the masks and improving laser engraving techniques, we analyze the quality of this DOE fabrication method by femtosecond laser recording. We compare the diffraction pattern of the masks manufactured with the simulated by Rayleigh-Sommerfeld method. Furthermore, we will study the effect of several processing parameters to increase the effectiveness of this fabrication technique.

Further author information: (Send correspondence to Angela Soria-Garcia)

Angela Soria-Garcia: E-mail: angsoria@ucm.es 


\section{METHODS}

\subsection{Design}

Several DOEs have been designed for different beam shape configurations, taking into account the laser processing capabilites. Far field DOEs have been designed using the Gerchberg-Saxton algorithm. On the other hand, when we want to visualise an intensity pattern in near-field, we implement the Fresnel integral.

These methods were implemented in the Python open source software module diffractio. ${ }^{12}$ The input arguments are the light beam, the desired intensity distribution in the image plane and the number of iterations, along propagation distance for far-field conditions. Nevertheles, for near field regime it is also necessary to indicate the DOE propagation distance. This method determines the amplitude or phase of the mask that provides the required irradiance distribution. Therefore, the output arguments are the DOE and the resulting error at each iteration. The algorithm allows us to analyse the behaviour of continuous or binary masks as well as amplitude or phase mask, which is important to know which type of masks are more efficient.

\subsection{Laser manufacturing process}

The designed DOEs have been fabricated using the direct writing technique with ultra-short laser pulses. The processing set up used is shown in Figure 1. It is formed by a $10 \mathrm{~W}$ Ytterbium-doped fiber laser, a power and beam size management module, a polarization management module, an XY scanner, an F-theta lens and a 3D motion stage. The femtosecond laser generates $280 \mathrm{fs}$ pulses at a central wavelength of $\lambda=1030 \mathrm{~nm}$.

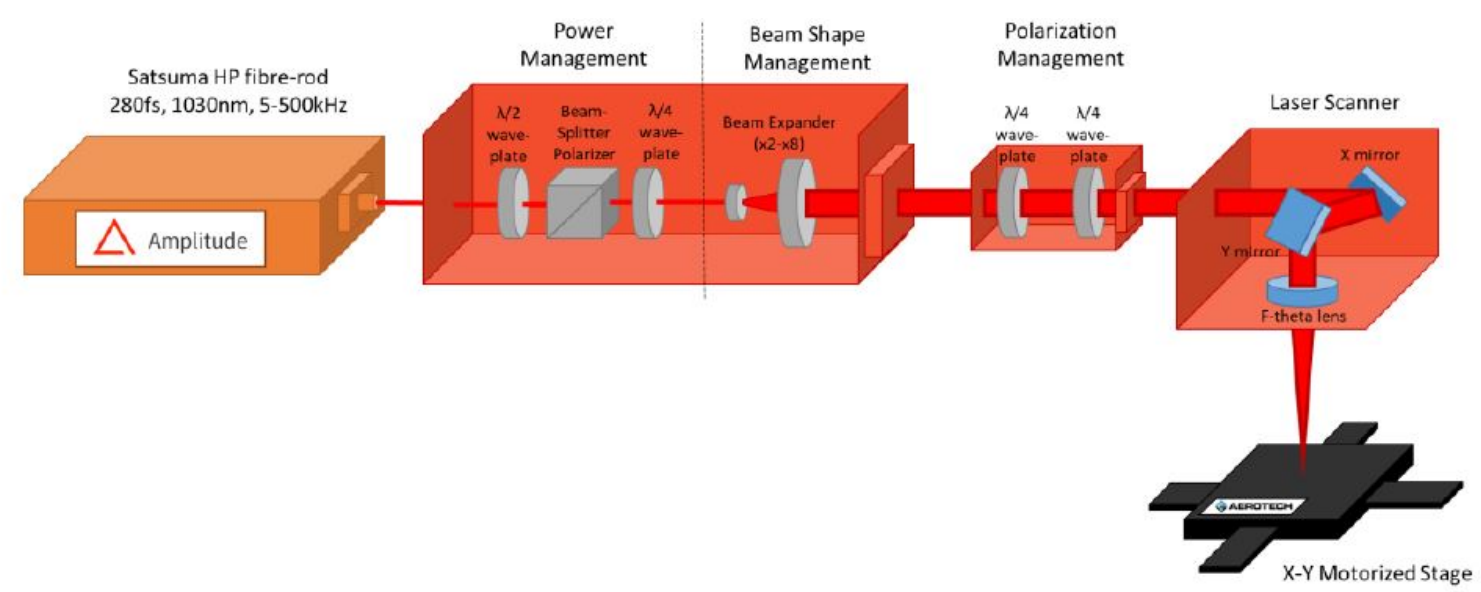

Figure 1: Set up used to manufacture diffractive optical elements.

In this work, we used standard $1 \mathrm{~mm}$ microscope slides as substrate and a platinum layer of around $200 \mathrm{~nm}$ has been placed on top. We removed this metal layer by laser ablation trying not to damage the glass. Therefore, we are able to create binary amplitude masks. Particularly, the pulse energy used in the mayority of the DOEs, was $12 \mu \mathrm{J}$. Figure 2 shows the fabricated masks, which have a pixel resolution of $10 \mu \mathrm{m} / \mathrm{px}$ and a surface area from $0.5 \mathrm{~cm}^{2}$ to $1 \mathrm{~cm}^{2}$.

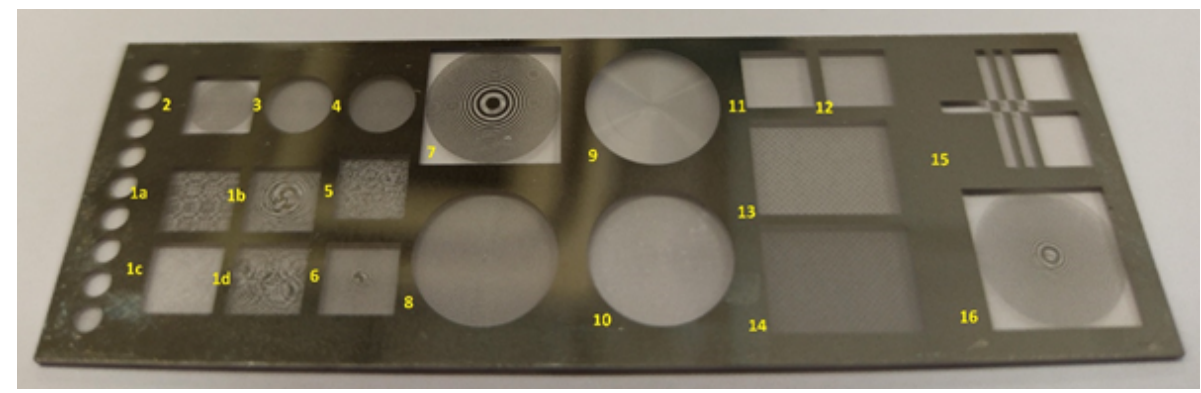

Figure 2: DOEs engraved using the femtosecond laser. 


\subsection{Diffraction pattern characterization}

First, we performed a dimensional analysis of the manufactured masks. For this purpose, we used a white light microscope (LEICA VMM 200) with x5 and x20 magnification and a 12 Mpx camera. The properties of the DOEs were characterized both in transmission and reflection configurations, as shown in Figures 3a and 3b respectively. In both cases, the light beam was provided by a He-Ne laser at $633 \mathrm{~nm}$. We used a polarizer to control the beam energy. A pinhole allowed clipping the beam in order to illuminate just one DOE at a time. We placed a lens ( $\mathrm{x} \mathrm{cm}$ focal length) after far-field DOEs so the mask and the camera (ImagingSource DMx 72BUC02 CMOS (resolution 2592x1944, 8 bits, $2.2 \mu \mathrm{m}$ square pixels)) were placed at the object and image focal planes respectively. We placed a 4 -f system between near-field DOEs and the camera in reflection configuration in order to be able to image the DOE and correctly measure the distance from it.

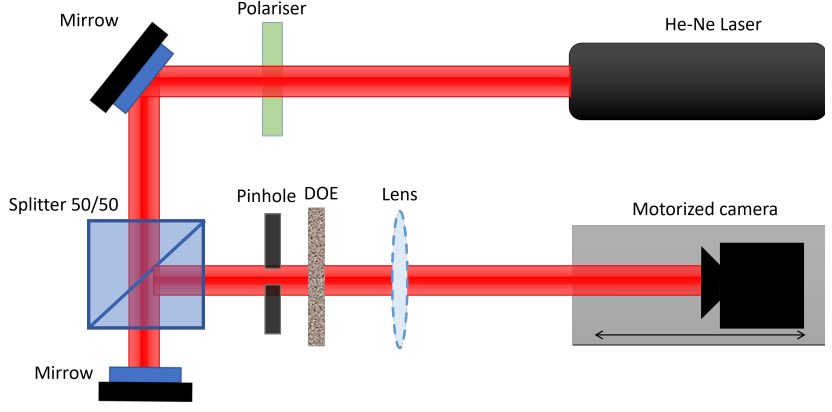

(a)

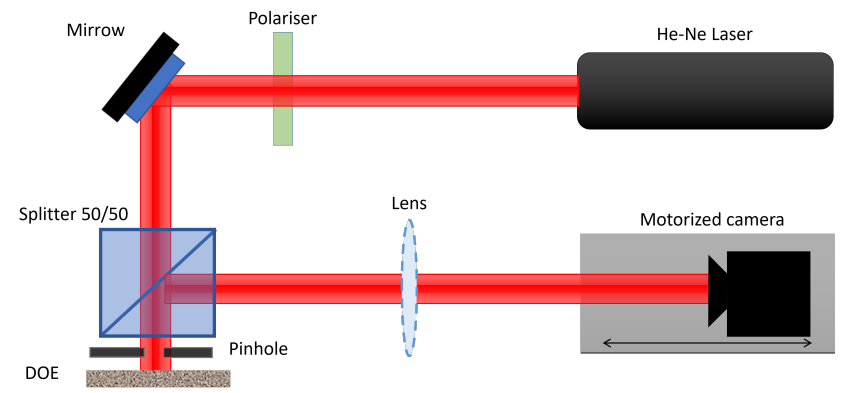

(b)

Figure 3: Set ups of the (a) transmission and (b) reflection configurations used to analyze the DOEs.

\section{RESULTS}

\subsection{Validation of the manufacturing process}

The fabricated DOEs have been observed with the microscope and photographed in order to compare their pattern with the design. With the aim of making an accurate comparison, we numerically binarized these pictures. As shown in Figures $4 \mathrm{a}$ and $4 \mathrm{~b}$, the results are satisfactory since their structure is adequately replicated. We also acquired an image of near-field DOE at the characterization setup (Figure 4c), furtherly validating the fabrication process.

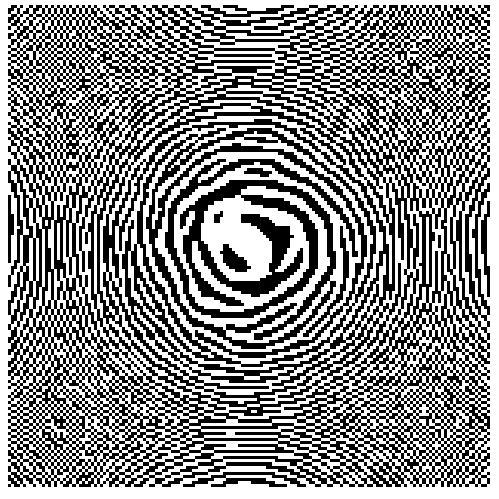

(a)

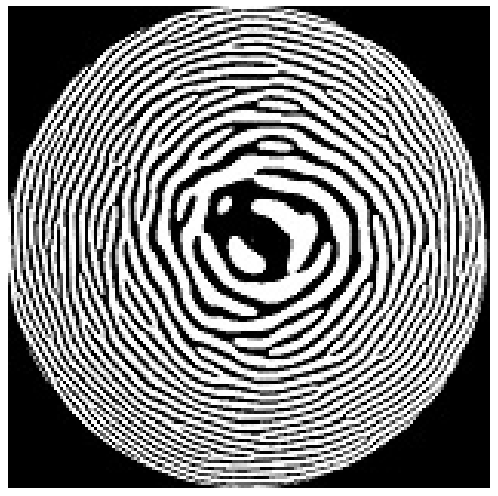

(b)

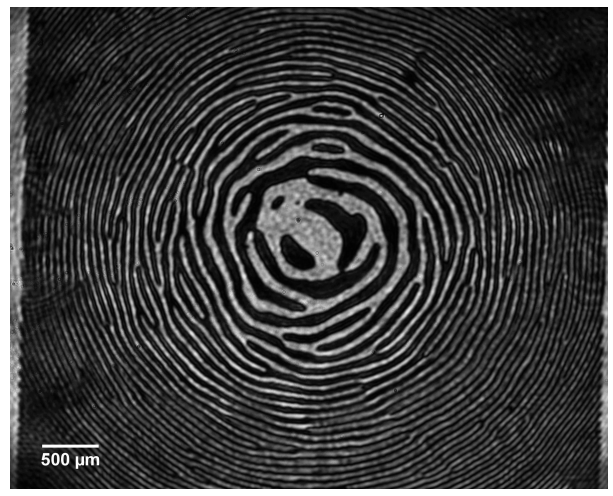

(c)

Figure 4: (a) Design of a near field DOE. Replicated mask observed (b) from the microscope and (c) from the characterization setup.

Although the manufacturing process was acceptable, some possible improvements have been identified. We recognized two main problems in the fabrication method: the pixelization and the incorrect pulse energy of the 
laser. On the one hand, when circular structures were manufactured, we observed that pixelization was excessive. We modified the fabrication setup in order to be able to reduce pixel size from 10 to $5 \mathrm{um}$ and fabricated an example DOE. The improvement was evident and it remains to analyze the optical quality of the results obtained.

On the other hand, we also analyzed the effect of the pulse energy in the quality of the DOEs in order to find the most suitable manufacturing parameters. Figure 5 shows several undesired results of the fabricated DOEs, which have been observed by optical microscopy. First, too high pulse energies might allow modification of the dielectric material (Figures 5a and 5b) due to nonlinear absorption effects, producing a modification of the refractive index. In fact, in Figure 5a we observed that the excessive intensity of irradiation has even caused small holes in the glass. Also, the pixel size depends on the pulse energy. In case of DOEs with great spatial variability a high or low dose will encrease or reduce the pixel size compared to the design value (Figures 5b and $5 \mathrm{c}$, respectively). As a consequence, the mask is not binarised correctly, altering the obtained result.

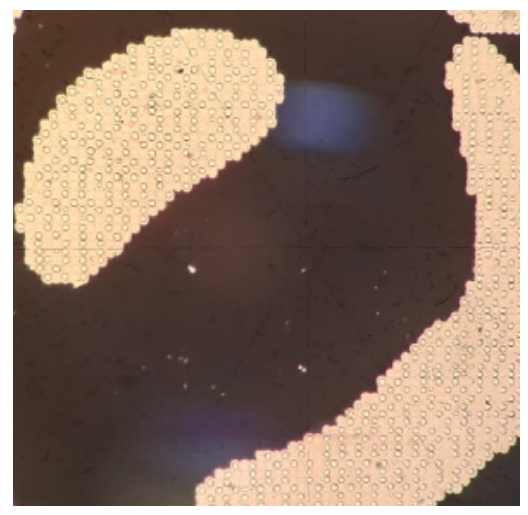

(a)

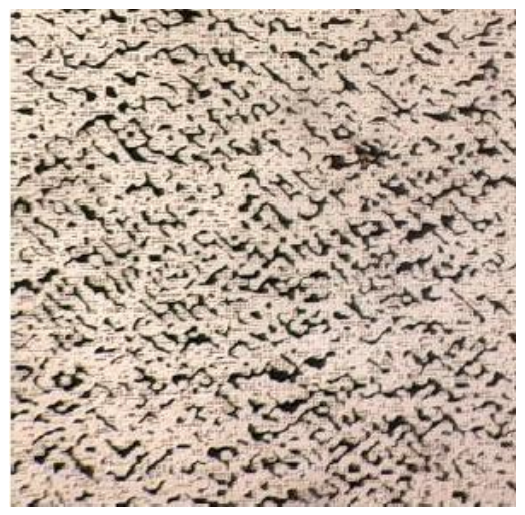

(b)

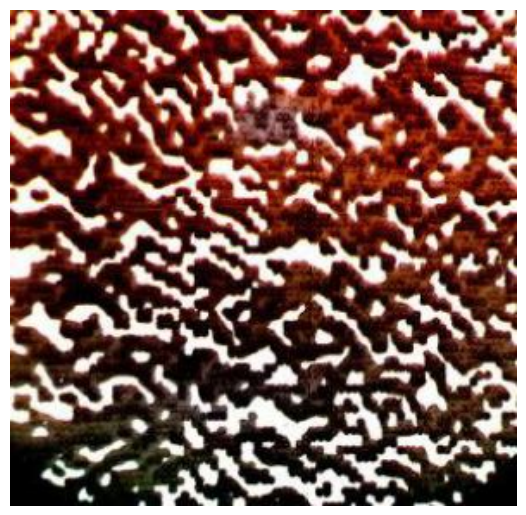

(c)

Figure 5: Optical microscopy images of DOEs where the pulse energy is (a)(b) excessive and (c) scarce.

\subsection{Analysis of the diffraction pattern}

We have analyzed several DOEs with different applications. First, we studied the behaviour of a far field mask in transmision configuration, whose objective is the formation of a specific image. ${ }^{13}$ Figure $6 \mathrm{a}$ and $6 \mathrm{~d}$ correspond with numerically simulated and experimental propagation of this DOE, which show that the theoretical irradiance distribution agrees with the experimental one.

Then, we focus on masks for beam shaping which are very useful in laser engraving field, as they direct the light towards a defined surface. ${ }^{14}$ Figure $6 \mathrm{~b}$ and $6 \mathrm{e}$ shows the result of the numerically simulated and experimental propagation of a near field multi-lens system in transmision configuration. As observed, the behaviour of this DOE is satisfactory and it focus the light on 16 different points. Other DOEs analyzed that have encouraging results are flat-top beamshaper (Figure 6c), which directs the light to a defined square. ${ }^{15}$ We have studied these masks in both far (transmision configuration) and near field regime (4-f reflection configuration), and its experimental behavior is in agreement with the simulated one (Figure 6f).

We have also analyzed whether it is better to work in near or far field regime. Although, in a first instance, it depends on one's own applications, we have observed that the near field mask presents more variability than the far field one. This is because the mask itself contains, in a certain sense, the lens necessary to form the image. However, the far field mask requires an additional lens on the set up, which does not take up much space. Therefore, these type of DOEs has the advantage of less variability.

Finally, it is worth noting the intensity of the $0^{\text {th }}$ diffraction order in Figures $6 \mathrm{~d}$ and $6 \mathrm{e}$. This light spot is always present in amplitude DOEs. Therefore, if we wanted to eliminate this diffraction order, we would have to fabricate phase masks. 


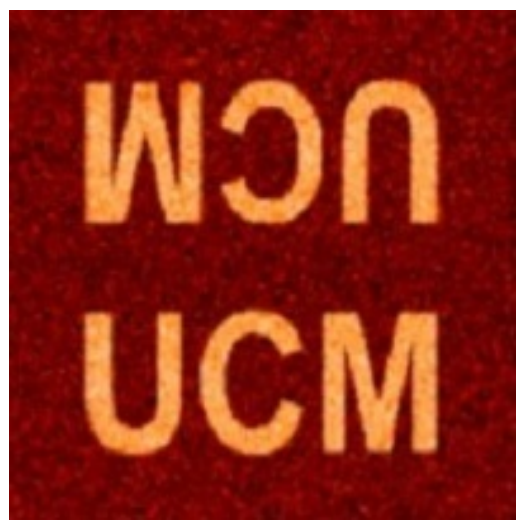

(a)

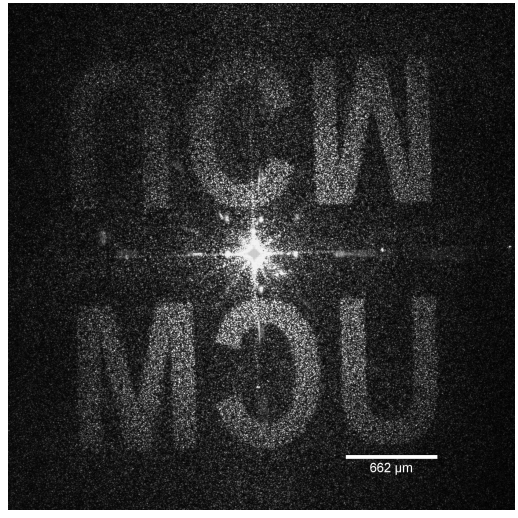

(d)

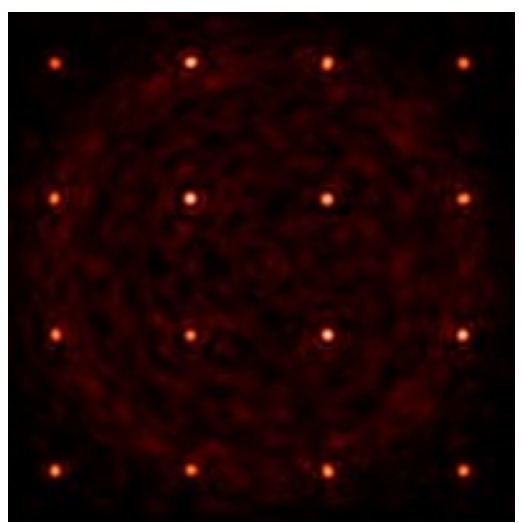

(b)

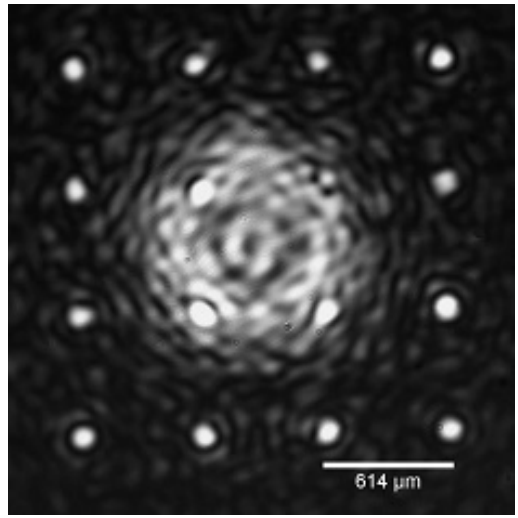

(e)

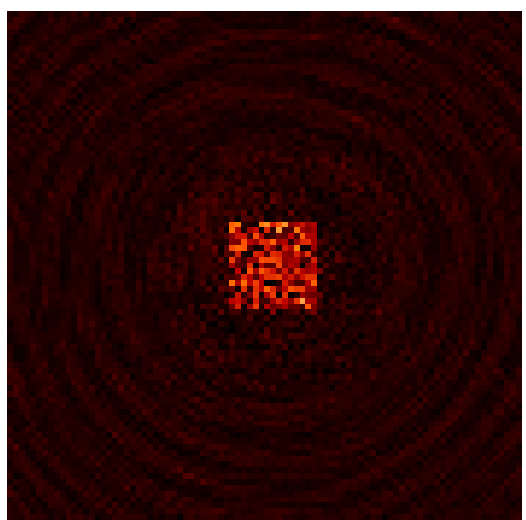

(c)

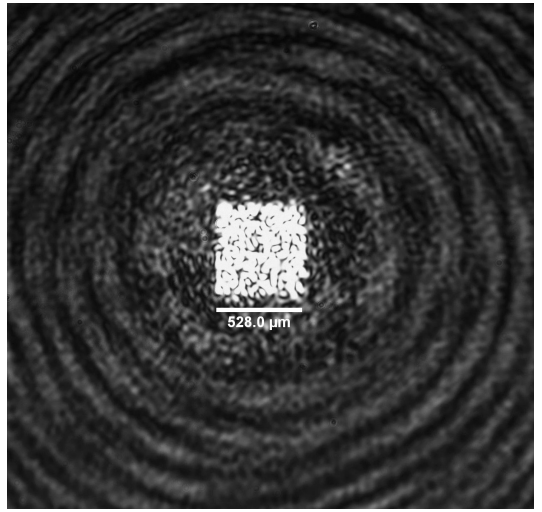

(f)

Figure 6: Diffraction pattern of three different DOEs obtained by simulation (up) and with the experimental setup (down).

\section{CONCLUSIONS}

In summary, we have found that the direct writing technique with ultra short laser pulses is valid for creating amplitude binary DOEs. In addition, with the experimental analysis, we have demonstrated the similarity between the computational and experimental results, proving that both the design and fabrication method are adequate. Although, the fabrication method has been excellent, some inaccuracies have been noted. We showed that it is important to control the irradiation dose of the material not to damage the glass.

\section{REFERENCES}

[1] Reichelt, S., Sahm, H., Leister, N., and Schwerdtner, A., "Capabilities of diffractive optical elements for real-time holographic displays," in [Practical Holography XXII: Materials and Applications], Bjelkhagen, H. I. and Kostuk, R. K., eds., 6912, 185 - 195, International Society for Optics and Photonics, SPIE (2008).

[2] Katz, S., Kaplan, N., and Grossinger, I., "Using diffractive optical elements," Laser Technik Journal 15(4), 29-32 (2018).

[3] Golub, M. A. and Grossinger, I., "Diffractive optical elements for biomedical applications," in [Biomedical Systems and Technologies II], Baldini, F., Croitoru, N. I., Dickinson, M. R., Frenz, M., Miyagi, M., Pratesi, R., and Seeger, S., eds., 3199, 220 - 231, International Society for Optics and Photonics, SPIE (1998).

[4] O'Shea, D. C., Suleski, T. J., Kathman, A. D., and Prather, D. W., [Diffractive Optics: Design, Fabrication, and Test (SPIE Vol. TT62)], SPIE- International Society for Optical Engineering (2003). 
[5] Veiko, V., Korolkov, V., Poleshchuk, A., Sinev, D., and Shakhno, E., "Laser technologies in micro-optics. part 1. fabrication of diffractive optical elements and photomasks with amplitude transmission," Optoelectronics, Instrumentation and Data Processing 53, 474-483 (09 2017).

[6] Gerchberg, R. W. and Saxton, W. O., "A practical algorithm for the determination of phase from image and diffraction plane pictures.," 35, 237-46 (1972).

[7] Sohn, J.-S., Lee, M., Kim, W.-C., Cho, E.-H., Kim, T.-W., Yoon, C.-Y., Park, N.-C., and Park, Y.P., "Design and fabrication of diffractive optical elements by use of gray-scale photolithography," Applied optics 44, 506-11 (03 2005).

[8] Nottola, A., Gerardino, A., Gentili, M., Di Fabrizio, E., Cabrini, S., Melpignano, P., and Rotaris, G., "Fabrication of semi-continuous profile diffractive optical elements for beam shaping by electron beam lithography," Microelectronic Engineering 53(1), 325-328 (2000).

[9] Caley, A. J., Braun, M., Waddie, A. J., and Taghizadeh, M. R., "Analysis of multimask fabrication errors for diffractive optical elements," Appl. Opt. 46, 2180-2188 (Apr 2007).

[10] Choi, J., Ramme, M., Anderson, T., and Richardson, M. C., "Femtosecond laser written embedded diffractive optical elements and their applications," in [Frontiers in Ultrafast Optics: Biomedical, Scientific, and Industrial Applications X], Heisterkamp, A., Neev, J., Nolte, S., and Trebino, R. P., eds., 7589, 199 - 205, International Society for Optics and Photonics, SPIE (2010).

[11] Roeder, M., Thiele, S., Hera, D., Pruss, C., Guenther, T., Osten, W., and Zimmermann, A., "Fabrication of curved diffractive optical elements by means of laser direct writing, electroplating, and injection compression molding," Journal of Manufacturing Processes 47, 402-409 (2019).

[12] Sanchez-Brea, L. M., "Diffratio, python module for diffraction and interference optics," https://pypi.org/project/diffractio/, (2019).

[13] Mikheev, G., Mikheev, K., and Anoshkin, I. E. A., "Laser images recording on aerosol-synthesized singlewalled carbon nanotube films," 887-890, Tech. Phys. Lett. 41 (2015).

[14] Mendivil, J., Hoyo, J., Solis, J., and Lifante, G., "Ridge waveguide laser in nd:linbo3 by zn-diffusion and femtosecond-laser structuring," Optical Materials 62 (12 2016).

[15] Fuse, K., "Beam shaping for advanced laser materials processing," Laser Technik Journal 12(2), 19-22 (2015). 